MATEC Web of Conferences 39, 01002 (2016)

DOI: $10.1051 /$ matecconf/20163901002

(C) Owned by the authors, published by EDP Sciences, 2016

\title{
Parametric design of silo steel framework of concrete mixing station based on the finite element method and MATLAB
}

\author{
Hui Long ${ }^{1}$, Changzheng Huang ${ }^{1, \mathrm{a}}$, Jing $\mathrm{Li}^{1}$ and Feng $\mathrm{Liu}^{1}$ \\ ${ }^{1}$ School of Physics and Mechanical \& Electrical Engineering, Shaoguan University, Shaoguan, China
}

\begin{abstract}
In most cases, the dimension parameters, shape parameters and position parameters of silo steel framework beams are changed as the productivity adjustment of the concrete mixing station, but the structure types of silo steel framework will remain the same. In order to acquire strength of silo steel framework rapidly and efficiently, it is need to provide specialized parametric strength computational software for engineering staff who does not understand the three-dimensional software such as PROE and finite element analysis software. By the finite element methods(FEM), the parametric stress calculation modal of the silo steel framework of concrete mixing station is established, which includes dimension parameters, shape parameters, position parameters and applied load parameters of each beams, and then the parametric calculation program is written with MATLAB. The stress equations reflect the internal relationship between the stress of the silo steel frames with the dimension parameters, shape parameters, position parameters and load parameters. Finally, an example is presented, the calculation results show the stress of all members and the size and location of the maximum stress, which agrees well with realistic cases.
\end{abstract}

\section{Introduction}

With the increase of the productivity capacity of the concrete mixing station, the silo in which the concrete materials are stored is getting larger and larger, and it brings increasingly extrusive the strength problems of the silo steel framework. The large deformation of the silo steel framework of the concrete mixing station is a commonly problem, which threats the safe operation of concrete mixing station[1]. At this moment, the strength of silo steel framework is mainly analyzed by the finite element method software such as ANSYS, ALGOR, ABQUS [2, 3, 4]. The finite element method, which can be discover the internal relationship between the strength of silo steel framework with dimension parameters, shape parameters, position parameters and load parameters, is widely used in static and dynamic modal of framed structures and shaft structure $[5,6]$. In almost all cases, the dimension parameters, shape parameters and position parameters of members of silo steel framework are changed as the productivity adjustment of the concrete mixing station when the structure of the silo steel framework of concrete mixing station is redesigned, but the structure types of silo steel framework will remain the same. In order to acquire strength rapidly and efficiently, it is need a parametric strength computational software for engineering staff, who does not understand the three-dimensional software such as PROE and finite element analysis software such as ANSYS etc. Based on the parametric strength computational software, all of the stress of the silo steel framework members is obtained by just entering the values of the applied load, beams shape, beams dimension and beams location. Up to date, there is no research material about building the parametric computational design of silo steel framework of concrete mixing station with finite element method and programed in MATLAB, which includes dimension parameters, shape parameters, position parameters and applied load parameters of each members.

This paper sets silo steel framework of concrete mixing station as a focus study. By finite element method (FEM), firstly is to establish a theoretical modal of the silo steel framework of concrete mixing station taking into account both load distribution and structure properties, including dimension parameters, shape parameters and position parameters of members of silo steel framework. According to the stress equations of the silo steel framework, a program is written with MATLAB language. Finally, an example is presented, the result show the stress of all members and the size and location of the maximum stress, which agrees well with realistic cases.

\footnotetext{
${ }^{\text {a }}$ Corresponding author: 422670309@qq.com
} 


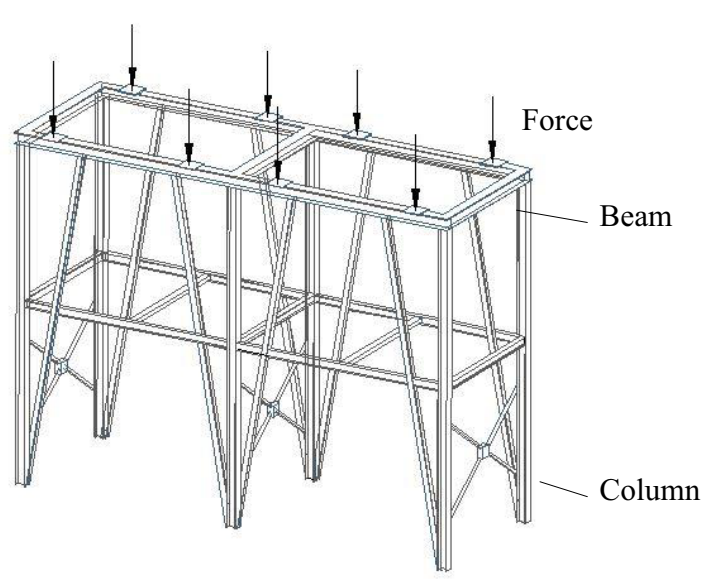

Figure 1. Silo steel framework of concrete mixing station

\section{Simplified analytical model of structure}

\subsection{Simplified plane model}

The structure of the silo steel framework of concrete mixing station and the applied force from silo stored the concrete material is shown in Fig.1. The structure of framework is, like clockwork along its longitudinal direction, collocated by a series beams and column beams. The loads acting upon the framework are usually uniform distributed along its longitudinal sense. Therefore, the portion between adjacent centroidal axes of two outer column beams could be extracted as the analytical element from the space structure, while the loads acting upon on the structure could be distributed to each analytical element by the longitudinal members. Because in each analytical element, its members as well as its loads lie in a single plane, the space structure can be simplified into the plane models shown as in Fig.2 [7].

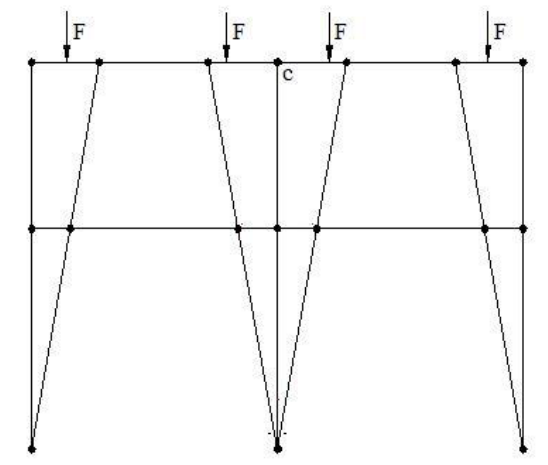

Figure 2. Calculation plane structure model

\subsection{Application of structural symmetry}

For even span steel frame, the node ${ }^{c}$ at the symmetric axis of structure has no antisymmetric horizontal displacement and rotary angle under the positive symmetric load. With the structure symmetry, half of the plan structure model substitute for the whole structure is used to calculate, shown in Fig.3.

\section{Beam element modal}

\subsection{Displacement function of beam element}

For a silo steel framework that is composed of the beam, all of the beams are tackled as beam element modal in this static analysis, shown in Fig.4. The elastic lateral displacement and longitudinal displacement of a point on neutral of beam $W(\bar{x}, t)$ and $V(\bar{x}, t)$ can be expressed as [8]:

$$
\begin{cases}W(\bar{x}, t)=\sum_{i} \phi_{i}(\bar{x}) u_{i}(t), & i=2,3,5,6 \\ V(\bar{x}, t)=\sum_{j} \phi_{j}(\bar{x}) u_{j}(t), & j=1,4\end{cases}
$$

Where, $\phi_{1}(\bar{x}), \phi_{2}(\bar{x}), \phi_{3}(\bar{x}), \phi_{4}(\bar{x}), \phi_{5}(\bar{x}), \phi_{6}(\bar{x})$ are the shape function; $\bar{x}$ is the location in the beam local coordinate system; $u_{1}, u_{4}$ are elastic longitudinal displacement at a node, $u_{2}, u_{5}$ are the elastic lateral displacement at a node; $u_{3}, u_{6}$ are the elastic rotation angles on axis at a node; That is, the displacement vector of beam element can be expressed as

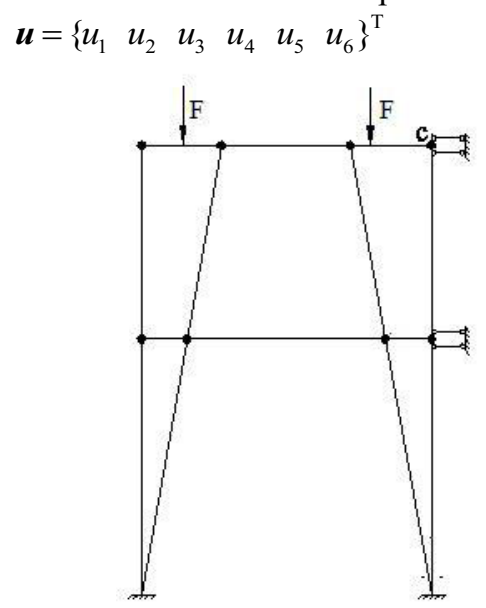

Figure 3. Simplified symmetry plane structure model

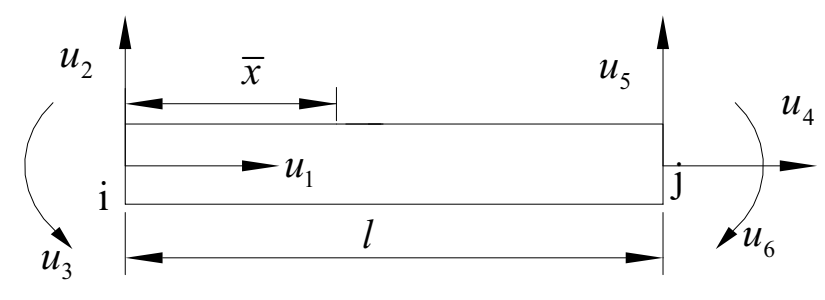

Figure 4. Beam element modal

\subsection{Stress-strain of beam element}

Based on the theory of elastic mechanics, the axial strain and bending strain of any point on cross section of beam element $\varepsilon_{\bar{x}}^{l}$ and $\varepsilon_{\bar{x}}^{b}$ can be expressed as:

$$
\varepsilon_{\bar{x}}^{l}=\frac{\partial V(\bar{x})}{\partial \bar{x}}
$$




$$
\varepsilon_{\bar{x}}^{b}=-\bar{y} \frac{\partial^{2} W(\bar{x})}{\partial \bar{x}^{2}}
$$

Where, $\bar{y}$ is the distance from any point on cross section to neutral axis $\bar{x}$.The strain of the beam element $\varepsilon_{x}$ is then written as

$$
\varepsilon_{\bar{x}}=\varepsilon_{\bar{x}}^{l}+\varepsilon_{\bar{x}}^{b}
$$

Eq.(5) can then be transformed as follows:

$$
\varepsilon_{x}=\boldsymbol{D} \boldsymbol{u}
$$

Where

$\boldsymbol{D}=\left[-\frac{1}{l}-\bar{y}\left(\frac{-6}{l^{2}}+\frac{12}{l^{3}}\right)-\bar{y}\left(\frac{-4}{l}+\frac{6}{l^{2}}\right) \frac{1}{l}-\bar{y}\left(\frac{6}{l^{2}}-\frac{12}{l^{3}}\right)-\bar{y}\left(\frac{-2}{l}+\frac{6}{l^{2}}\right)\right]$

$\boldsymbol{D}$ is the strain transformation matrix of beam element

The stress-strain relationship in the elastic range can be written as

$$
\boldsymbol{\sigma}_{\bar{x}}=E \boldsymbol{\varepsilon}_{x}
$$

Where $E$ is the elasticity modulus of the beam element.

\subsection{Static equation of beam element}

Applying the virtual work principle equation $\left(\left\{\boldsymbol{\delta}^{*}\right\}^{e}\right)^{\mathrm{T}}\{\overline{\boldsymbol{f}}\}=\left(\left\{\boldsymbol{\delta}^{*}\right\}^{e}\right)^{T} \iiint_{v}[\boldsymbol{D}]^{T} E[\boldsymbol{D}] \mathrm{d} x \mathrm{~d} y \mathrm{dz}\{\boldsymbol{u}\}$

Where $\left\{\delta^{*}\right\}^{e}$ is the node virtual displacement as a random value. Eq.(8) can then be transformed as follows:

$$
\{\overline{\boldsymbol{f}}\}^{e}=\iiint_{v}[\boldsymbol{D}]^{T} E[\boldsymbol{D}] \mathrm{d} x \mathrm{~d} y \mathrm{dz}\{\boldsymbol{u}\}
$$

Defining the beam element stiffness matrix $[\overline{\boldsymbol{k}}]^{e}=\iiint_{v}[\boldsymbol{D}]^{T} E[\boldsymbol{D}] \mathrm{d} x \mathrm{dydz}$, Eq.(9) can be written as

$$
\{\overline{\boldsymbol{f}}\}^{e}=[\overline{\boldsymbol{k}}]^{e}\{\boldsymbol{u}\}
$$

\section{Mathematic modeling of silo steel framework of concrete mixing}

The study focus is shown as Figure 5, the simplified symmetry plane structure of silo steel framework is divided into $n$ beam element by finite element method (FEM). The coordinate relationship of $i$ th beam element from local coordinate system to global coordinate system is transformed as follows [9]

$$
\boldsymbol{u}_{i}=\boldsymbol{R}_{i} \boldsymbol{U}_{i}^{e}
$$

Where $\boldsymbol{R}_{i}$ is the coordinate transformation matrix from local coordinate system to global coordinate system.

Substituting Eq.(11) into Eq.(10), the static equation of $i$ th beam element in global coordinate system is expressed as

$$
\{\boldsymbol{f}\}_{i}^{e}=\left[\boldsymbol{k}_{i}^{e}\right] \boldsymbol{U}_{i}^{e}
$$

Where $\boldsymbol{k}_{i}^{e}=\boldsymbol{R}_{i}^{T} \overline{\boldsymbol{k}}_{i} \boldsymbol{R}_{i}, \boldsymbol{f}_{i}^{e}=\boldsymbol{R}_{i}^{T} \overline{\boldsymbol{f}}_{i} \boldsymbol{R}_{i}$. The numbering relati onship of $i$ th beam element from local numbering to global numbering is defined as [9]:

$$
\boldsymbol{U}_{i}^{e}=\boldsymbol{B}_{i} \boldsymbol{U}
$$

Where $\boldsymbol{B}_{i}$ is the numbering transformation matrix from local numbering to global numbering, $\boldsymbol{U}$ is the generalized displacement vectors in the global system.
Appling the Eq.(13) into Eq.(12), the static equation of $i$ th beam element in global coordinate system and global numbering is written as

$$
\boldsymbol{F}_{i}^{e}=\boldsymbol{K}_{i}^{e} \boldsymbol{U}
$$

Where, $\boldsymbol{K}_{i}^{e}=\boldsymbol{B}_{i}^{T} \boldsymbol{R}_{i}^{T} \boldsymbol{k}_{i}^{e} \boldsymbol{R}_{i} \boldsymbol{B}_{i}, \quad \boldsymbol{F}_{i}^{e}=\boldsymbol{B}_{i}^{T} \boldsymbol{R}_{i}^{T} \overline{\boldsymbol{f}}_{i}^{e} \boldsymbol{R}_{i} \boldsymbol{B}_{i}$.

Assembling all the static equation of beam elements with global numbering in global coordinate system yields the following static equation of the silo steel system

$$
\boldsymbol{F}=\boldsymbol{K} \boldsymbol{U}
$$

Where, $\boldsymbol{K}=\sum_{i=1}^{n} \boldsymbol{K}_{i}^{e}, \boldsymbol{F}=\sum_{i=1}^{n} \boldsymbol{F}_{i}^{e}, \boldsymbol{F}$ contains only external force as the interact force between the elements is counteracting.

Substituting Eqs.(13),Eq.(11),and $\mathrm{Eq}(7)$ into Eq (15) and applying the strain displacement relation $\boldsymbol{\varepsilon}_{i}=\boldsymbol{D}_{i} \boldsymbol{u}_{i}[8]$ yields the following stress equation of $i$ th beam element can be written as follows

$$
\boldsymbol{\sigma}_{i}=E \boldsymbol{D}_{i} \boldsymbol{R}_{i} \boldsymbol{B}_{i} \boldsymbol{K}^{-1} \boldsymbol{F}
$$

Where, $\boldsymbol{K}$ is related to the member dimension parameters and shape parameters, $\boldsymbol{R}_{i}$ is related to the members location parameters, and $\boldsymbol{F}$ is related to the applied load parameters of the silo steel framework. One is should note that Eq. (16), the right side of which includes dimension parameters, shape parameters, position parameters and applied load parameters of each members, each member stress $\sigma_{i}$ on the left side can then be obtained by applying all the parameters on the right side of the equation. Namely, the stress of the silo steel frames can be obtained.

\section{Number validation}

The simplified plane schematic of the silo steel frame is composed of eight steel beams as shown in Fig.5. Each member of silo steel frame is composed of structure carbon steel $\mathrm{H}$ beam profile, the elastic modules is $206 \mathrm{GPa}$, the density is $7.85 \mathrm{Kg} / \mathrm{m}^{3}$, the Poisson's ration is 0.26 . In general, the simplified plane steel frame is divided into sixteen elements, The physic parameters of each element is shown in Table 1.

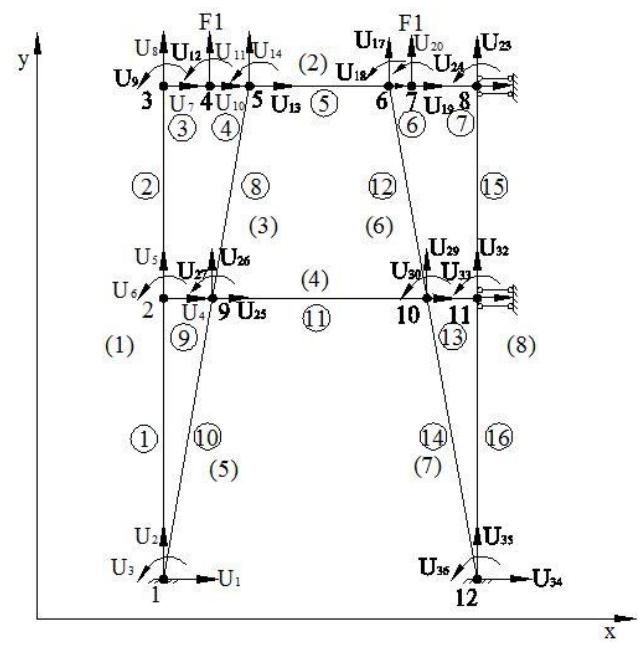

Figure 5. Finite element model of the silo steel frame 
Table 1. The parameters of each element

\begin{tabular}{|c|c|c|c|c|c|}
\hline No. & $\begin{array}{c}\text { Steel } \\
\text { Type }\end{array}$ & $\begin{array}{c}\text { Cat } \\
\text { egor } \\
\mathbf{y}\end{array}$ & $\begin{array}{c}\text { Length } \\
/ \mathbf{m m}\end{array}$ & $\begin{array}{c}\text { Size } \\
\text { (Height } \times \\
\text { Width) } \\
\mathbf{m m} \times \mathbf{m m}\end{array}$ & $\begin{array}{c}\text { Angle } \\
\text { between } \\
\text { beam axis } \\
\text { and } \\
\text { axis } x /{ }^{\circ}\end{array}$ \\
\hline 1 & H & HW & 5380 & $250 \times 250$ & 90 \\
\hline 2 & H & HW & 4060 & $250 \times 250$ & 90 \\
\hline 3 & H & HW & 880 & $300 \times 300$ & 0 \\
\hline 4 & H & HW & 704 & $300 \times 300$ & 0 \\
\hline 5 & H & HW & 2725 & $300 \times 300$ & 0 \\
\hline 6 & H & HW & 416 & $300 \times 300$ & 0 \\
\hline 7 & H & HW & 1256 & $300 \times 300$ & 0 \\
\hline 8 & H & HM & 3864 & $200 \times 150$ & 261 \\
\hline 9 & H & HM & 944 & $200 \times 150$ & 180 \\
\hline 10 & H & HM & 4860 & $200 \times 150$ & 259 \\
\hline 11 & H & HM & 4085 & $200 \times 150$ & 0 \\
\hline 12 & H & HM & 3864 & $200 \times 150$ & 279 \\
\hline 13 & H & HM & 992 & $200 \times 150$ & 0 \\
\hline 14 & H & HM & 4860 & $200 \times 150$ & 281 \\
\hline 15 & H & HW & 4060 & $300 \times 300$ & 270 \\
\hline 16 & H & HW & 5380 & $300 \times 300$ & 270 \\
\hline
\end{tabular}

Let $U_{1}, U_{4}, \ldots, U_{34}$ be the displacement vector along the axis $x$ at each node; $U_{2}, U_{5}, \ldots, U_{35}$ is the displacement vector along the axis $y$ at each node; $U_{3}, U_{6}, \ldots, U_{36}$ is the rotation angles at each node.

The force applied at the node 4 and node 7 is $784 \mathrm{KN}$, which is shown in Fig.5.Applying the fixed boundary conditions at node 1 and node 12, taking the lateral displacement constraint at node 8 and node 2 .

The stress curve of the silo platform beam member (2) obtained by parametric calculation program with MATLAB language is shown in Fig.6. As can be seen, the maximum stress is at the left side forced location, which is also the position of node 4 , and the maximum stress is $195 \mathrm{Mpa}$, which agrees well with realistic cases.

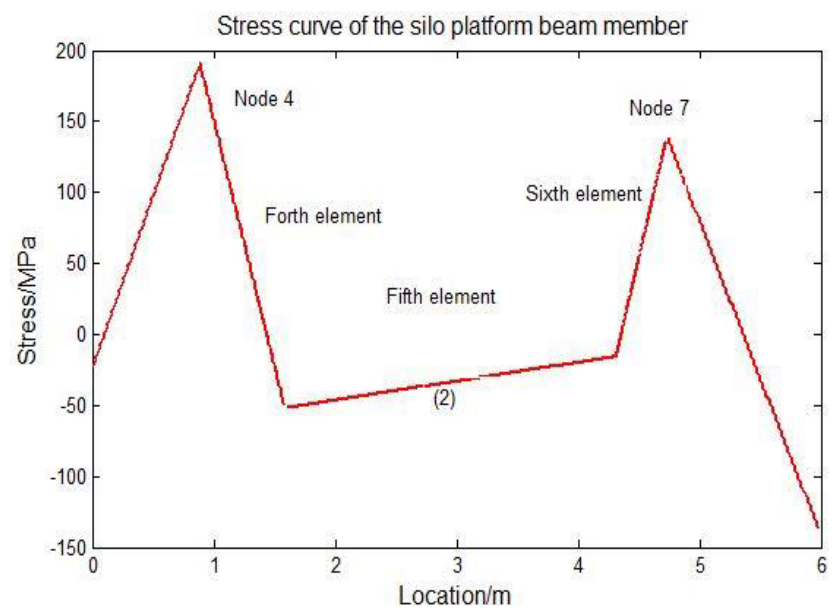

Figure 6. The stress distribution of the platform beam member

\section{Conclusions}

First, the parametric stress calculation modal, which is established by finite element method, can clearly show the relations between the stress of the silo steel frames and the member parameters such as shape parameter, dimension parameter and the location parameter.

Second, the corresponding parametric calculation program written with MATLAB language can quickly show the stress of all members and the size and location of the maximum stress when the shape parameter, dimension parameter and location parameter is changed, which will be easy for strength analysis and possible to realize the strength analysis of the special structure in certain software

On the other hand, the analysis method may provide the guidance for similar structure.

\section{Acknowledgements}

This work was financially supported by the Guangdong science and technology planning project (2012B091100 199, 2012KJCX0095), the Foundation for Distinguished Young Talents in Higher Education of Guangdong (Yq2014156).

\section{References}

1. C. Topkaya, J. Michael Rotter, Ring Beam Stiffness Criterion for Column-Supported Metal Silos, Journal of engineering mechanics,137,12 ( 2011)

2. M. Wojcik, M. Jecmenica. Numerical Calculations of Wall Pressures and Stresses in Steel Cylindrical Silos with Concentric and Eccentric Hoppers, Particulate Science and Technology,21(2003)

3. M.T. Abdel-Fatth, I. D. Moore, T.T. Abdel-Fatth, Behavior of elevated concrete silos filled with saturated solids, Can. J. Civ. Eng, 33(2006)

4. J. F. Chen, S. K. Yu, J.Y. Ooi, J. M. Rotter, Finite-Element Modeling of Filing Pressures in a full-scale silo, Journal of engineering mechanics, 127,10 (2001)

5. D. Guines, E. Ragneau, B. Kerour. 3D finite-element simulation of a square silo with flexible walls, Journal of engineering mechanics . Vol.127,10 (2001)

6. K. H. Lien, Y. J. Chiou, P. A. Hsiao, Vector Form Intrinsic Finite Element Analysis of Steel Frames with Semi-rigid Joints, Journal of Structural Engineering,138, (2012)

7. S. H Bao, Y. Q. Gong, Structure Mechanics, (Wuhan university technology press, Wuhan, 2005)

8. O.C. Zienkiewic, R.L Taylor, The finite method.,5th ed.,vol.1. (Mcgraw-hill press, New York, 2000)

9. G.. W. Cai , R. G. Wang, Z. J. Li, The dynamic equation and simulation of a linkage mechanism fabricated from three-dimensional braided composite materials, JSME International Journal, Series C, 49 (2006) 